\title{
A Biomechanical Comparison of Locked Plate Fixation With Percutaneous Insertion Capability Versus the Angled Blade Plate in a Subtrochanteric Fracture Gap Model
}

\author{
Brett D. Crist, MD, Afshin Khalafi, MD, Scott J. Hazelwood, PhD, and Mark A. Lee, MD
}

\begin{abstract}
Objectives: The angled blade plate has been the historical standard in fixed-angle extramedullary subtrochanteric femur fracture fixation, but it requires an extensile lateral approach to the femur. Little formal evaluation exists for specifically designed percutaneous extramedullary implants. The purpose of this study was to compare 3 locked plating constructs, all with percutaneous insertion capability, with the standard 95-degree angled blade plate to determine whether specifically designed fixed-angle extramedullary implants for subtrochanteric femur fractures were biomechanically comparable to the angled blade plate.
\end{abstract}

Methods: Forty composite adult femurs were divided into 4 equal groups. The constructs evaluated included a 95-degree angled blade plate, a broad 4.5-mm combination locking plate, and a precontoured proximal femoral locking plate (PFLP) with and without an oblique, angled strut or "kickstand" screw. A 30-degree wedge osteotomy was used to create a subtrochanteric fracture gap model. Each specimen underwent axial and torsional stiffness testing along with cyclic axial loading to failure.

Results: Axial stiffness testing revealed that the PFLP with the "kickstand" screw was the stiffest construct $(92.2 \pm 17.4 \mathrm{Nm} / \mathrm{m})$, which was $211 \%$ stiffer than the blade plate, $309 \%$ stiffer than the broad plate, and 194\% stiffer than the PFLP without the kickstand screw. The blade plate had the highest torsional stiffness $(2.42 \pm 0.08$ $\mathrm{Nm} /$ degree), which was $151 \%$ stiffer than the broad plate, $128 \%$ stiffer than the PFLP with the kickstand, and $138 \%$ stiffer than the PFLP without the kickstand screw. The PFLP with the kickstand screw had the least irreversible deformation $(6.3 \mathrm{~mm})$, which was $52 \%$ less than the broad plate and $61 \%$ less than the PFLP without the kickstand screw.

Conclusions: Our data reveal that the PFLP with the "kickstand" screw provides more axial stiffness, less torsional stiffness, and equivalent irreversible deformation to cyclic axial loading when compared with the blade plate.

Key Words: subtrochanteric femur fractures, locking plates, biomechanical study

\section{INTRODUCTION}

Subtrochanteric femur fractures in young patients are high-energy injuries that require durable implants to maintain stability over potentially extended healing times. Although intramedullary fixation has a proven track record for subtrochanteric femur fractures and is probably the current gold standard, ${ }^{1-12}$ some surgeons prefer extramedullary fixation and have used it successfully. ${ }^{1,13-27}$ As implants and techniques continue to evolve, particularly with the use of locking plates and minimally invasive plating, extramedullary devices may have a more significant role in the treatment of subtrochanteric femur fractures.

There are advantages and disadvantages to both intramedullary and extramedullary devices. Due to the technically challenging deformities encountered in subtrochanteric femur fractures, both techniques may require an open reduction if closed reduction techniques have failed. However, open reduction can be performed without significantly devitalizing the fracture fragments. Biomechanical studies that have compared intramedullary and extramedullary devices have demonstrated that intramedullary fixation, due to its loadsharing properties, has greater axial ${ }^{4,5}$ and bending stiffness, ${ }^{4}$ but less torsional control ${ }^{4}$ in simulated subtrochanteric femur fractures. Because of its relative stability, intramedullary fixation has been shown to have significantly more interfragmentary motion at the fracture site when compared with extramedullary fixation. ${ }^{15}$ One of the greatest benefits of intramedullary fixation is that it creates an internal buttress preventing medial femoral shaft translation, which decreases the risk of fixation failure and fracture collapse. ${ }^{2,5,19}$ Hence, there has been a lower rate of reoperations reported, due to lower rates of implant failure and nonunion, in some clinical trials that have compared intramedullary and extramedullary devices. ${ }^{6,8,10,14,28,29}$

Although closed intramedullary nailing techniques have been successful in treating subtrochanteric femur fractures and have allowed for nail insertion via a small access incision, there are disadvantages to this technique. Significant insertion 
site morbidity has been reported including trochanteric pain, abductor weakness, and heterotopic bone formation. ${ }^{13,14,30,31}$ Furthermore, intramedullary fixation transiently ablates the endosteal blood supply, ${ }^{21,32}$ is technically demanding with proximal fracture extension involving the nail insertion site, ${ }^{3,17,21,33}$ can lead to iatrogenic fracture or propagation of the fracture, ${ }^{31,34,35}$ and has a concerning risk of varus malunion, if not correctly addressed intraoperatively. ${ }^{29,36,37}$

A wide variety of extramedullary implants have been used for the treatment of subtrochanteric femur fractures over the years and each has specific advantages and disadvantages. Currently, the most likely reason to use an extramedullary device is in subtrochanteric femur fractures that have extension into the nail entry site..$^{3,9,17,21,33}$ Fixed-angle implants have been evaluated in both clinical and biomechanical studies. ${ }^{1,13-21}$ The angled blade plate is considered by some to be the gold standard in extramedullary fixed-angle proximal femoral plate fixation, but it requires an extensile lateral approach to the femur for insertion. ${ }^{7,18,20,28,37}$ In an attempt to minimize soft tissue dissection and optimize the biological healing potential of plated fractures, open plating with indirect reduction techniques and submuscular plating with indirect reduction techniques for the proximal and distal femur and the femoral shaft have become increasingly popular. ${ }^{7,8,18,20,21,37-39}$ Although minimally invasive techniques have been developed for proximal femoral fractures using traditional extramedullary implants, there has been little formal evaluation of specifically designed percutaneous stabilization devices, specifically locked plates, for the proximal femur. ${ }^{8,21,39}$ The purpose of this study was to compare the axial and torsional stiffness and cyclic loading with failure properties of 3 locked plating constructs with percutaneous insertion capability to the standard 95-degree angled blade plate.

\section{MATERIALS AND METHODS}

A total of 40 third-generation composite adult femurs (Sawbones; Pacific Research Laboratories, Vashon, WA) were obtained and randomly divided into 4 groups, each with 10 specimens (Table 1). Third-generation composite femurs were used because of their similar biomechanical properties to human cadaveric specimens and decreased specimen variability. ${ }^{40,41}$ The implants used in all 4 groups were made by the same manufacturer for consistency, both with plate material used and with screw design (Synthes, USA, Paoli, PA). Group 1 (control) consisted of femurs plated with the 95-degree angled blade plate. Group 2 (broad locking compression plate) consisted of femurs stabilized with a contoured broad $4.5-\mathrm{mm}$ locking compression plate. Group 3 [proximal femoral locking plate (PFLP) with kickstand] femurs were stabilized with a precontoured PFLP including an oblique, locking strut or "kickstand" screw. Group 4 (PFLP without kickstand) used the same PFLP minus the "kickstand" screw. After standard plate positioning and application, a 30-degree wedge osteotomy was created at the level of the lesser trochanter to create a subtrochanteric gap model using a previously described technique. ${ }^{42}$ This osteotomy creates an unstable fracture similar to an OTA 31A3, 32B1.1, C1.1, B2.1, C2.1, $\mathrm{B} 3.1$, and $\mathrm{C} 3.1 .^{43}$ Although we acknowledge that fracture reduction is a key component for construct stiffness in the clinical situation, the osteotomy was created after implant application to allow for reproducible placement of the implants without radiographic assistance and to reduce the variability of fracture reduction and its effect on testing. Radiographs of each construct group after osteotomy is shown in Figure 1.

The load was applied to the head of the femur through a custom mold. The condyles of the distal end of the femur were also held in a custom mold that was secured to the materials testing machine. The specimens were supported in the testing machine by a ball bearing to avoid uncontrolled torque or bending similar to that previously described by Cordey et al. ${ }^{44}$ Each specimen underwent axial and torsional stiffness testing along with cyclic axial loading to failure to determine irreversible deformation. All testing was performed using an Instron 5800R (Instron, Canton, MA) materials testing machine. The testing apparatus for axial stiffness and cyclic axial loading to failure protocols is shown in Figure 2. The line of action of the axial force went through the femoral head proximally and through the intercondylar notch distally to simulate physiologic loading in single-leg stance along the mechanical axis. Axial stiffness testing was performed first and consisted of loading each specimen to $500 \mathrm{~N}$ at a rate of $5 \mathrm{~mm} / \mathrm{min}$.

Before cyclic axial loading to failure, torsional stiffness testing (Fig. 3) was performed. The proximal end was held in a custom mold and the distal end was secured in a chuck. Precise positioning was done to ensure that the femoral axis was aligned with the axis of rotation. The custom proximal fixture was mounted to a bearing system. The Instron imparted a force to a lever attached to the bearing to allow rotation of the

TABLE 1. Pertinent Plate Design Characteristics

\begin{tabular}{|c|c|c|c|c|}
\hline Group & Plate Type & Plate Length (Hole) & Proximal Fixation & Shaft Fixation \\
\hline 1 & 95-degree blade plate (control) & 14 & 70-mm blade & 36- and 4.5-mm cortical screws \\
\hline 2 & Broad 4.5-mm LCP & 14 & Two $80-$ and $5.0-\mathrm{mm}$ solid locking screws & $\begin{array}{l}\text { 36- and 5.0-mm solid bicortical } \\
\text { locking screws }\end{array}$ \\
\hline 3 & PFLP with kickstand & 8 & $\begin{array}{l}\text { - 90-mm (hole 1) and } 75 \mathrm{~mm} \text { (hole 2) 7.3-mm } \\
\text { cannulated proximal locking screws } \\
\text { 85-mm (hole 3) 5.0-mm cannulated } \\
\text { "kickstand" locking screw }\end{array}$ & $\begin{array}{l}\text { 36- and 5.0-mm solid bicortical } \\
\text { locking screws }\end{array}$ \\
\hline 4 & PFLP without kickstand & - & $\begin{array}{l}\text { - 90-mm (hole 1) and 75-mm (hole 2) } 7.3-\mathrm{mm} \\
\text { cannulated proximal locking screws }\end{array}$ & $\begin{array}{l}\text { 36- and } 5.0-\mathrm{mm} \text { solid bicortical } \\
\text { locking screws }\end{array}$ \\
\hline
\end{tabular}




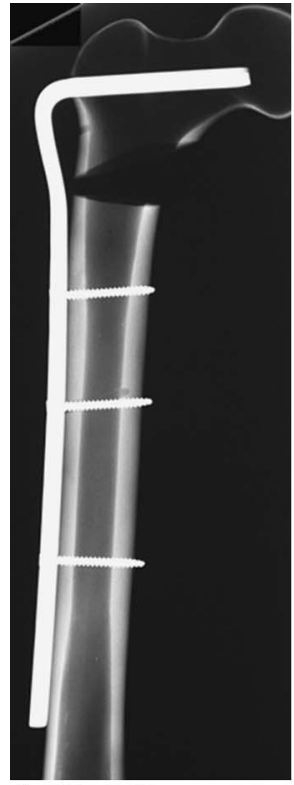

A Blade Plate

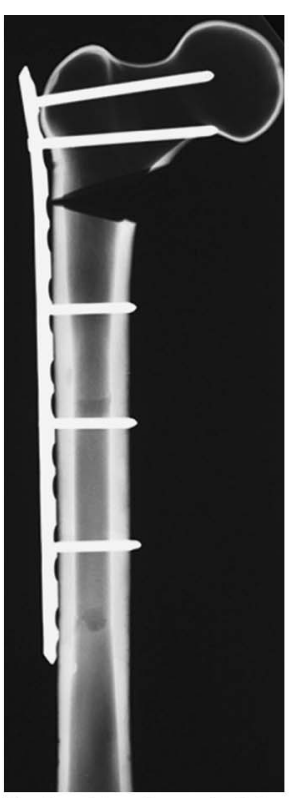

B Locking broad plate

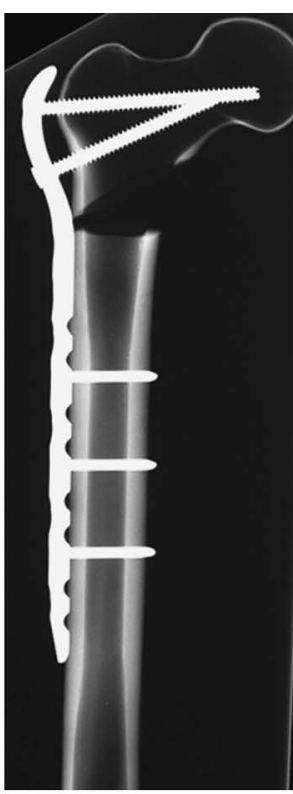

C PFLP w/o kickstand

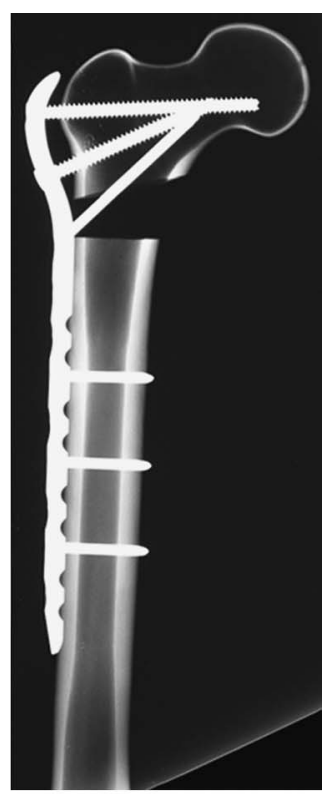

PFLP with kickstand femur about its longitudinal axis. The torsional cycle was applied in an external rotation direction based on the anatomy of the femoral model. External rotation was chosen because it is the typical rotational deforming force on the proximal femur associated with a subtrochanteric fracture. Torsional loading was performed by preloading each specimen to $5 \mathrm{Nm}$ of torque and then loading the specimen to a maximum torque of $20 \mathrm{Nm}$ at a rate of 25 degrees $/ \mathrm{min}$.

Cyclic axial loading to failure was performed last with a preload of $100 \mathrm{~N}$, and each specimen undergoing 10 cycles at each peak load from 300 to $1000 \mathrm{~N}$ in $100-\mathrm{N}$ increments at a rate of $0.75 \mathrm{~mm} / \mathrm{s}$ as previously described for the distal femur. ${ }^{45,46}$ Each load cycle was conducted as a ramp load in displacement control at a rate of $0.75 \mathrm{~mm} / \mathrm{s}$ to the peak load of the cycle. The construct was considered to have failed if the implant or femur model fractured, if the implant pulled out of the femur, if the medial edges of the osteotomy contacted, or if there was irreversible deformation present on completion of the cyclic axial loading protocol.

Axial stiffness and torsional stiffness were calculated from the linear portion of the load-displacement curve and torque-angle graph, respectively. For cyclic axial loading to failure, a time-displacement curve was created (Fig. 4). ${ }^{47}$ The irreversible deformation was determined by subtracting the amount of displacement present at the beginning of the $300 \mathrm{~N}$ cycle from the displacement after the final cycle.

Data were analyzed by using a 1-way analysis of variance, and Fischer post hoc least significant difference criterion was used to correct for multiple group comparisons. $P$ values less than 0.05 were considered indicative of significant differences.

\section{RESULTS}

Axial stiffness testing revealed significant differences between the 4 different construct groups (Table 2). The PFLP with kickstand $(92.2 \pm 17.4 \mathrm{Nm} / \mathrm{m})$ construct was axially stiffer than all of the other constructs. It was $211 \%$ stiffer than the blade plate construct $(P<0.001), 309 \%$ stiffer than the broad plate construct $(P<0.001)$, and $194 \%$ stiffer than the PFLP without kickstand construct $(P<0.001)$. The only other significant difference was that the PFLP without kickstand construct was $106 \%$ stiffer than the broad plate construct $(P=$ $0.0301)$.

The blade plate group $(2.42 \pm 0.08 \mathrm{Nm} /$ degree $)$ had significantly more torsional stiffness than all the other constructs. It was $151 \%$ stiffer than the broad plate group $(P<0.0001), 128 \%$ stiffer than the PFLP with the kickstand group $(P<0.0017)$, and $138 \%$ stiffer than the PFLP without the kickstand screw group $(P=0.002)$. There were no other statistically significant differences between construct groups.

The results of cyclic axial loading to failure demonstrate a variety of differences between the groups. The timedisplacement curve (Fig. 4) enabled calculation of the irreversible deformation that occurred. The PFLP with kickstand construct $(6.3 \pm 1.2 \mathrm{~mm})$ had $52 \%$ less irreversible deformation than the broad plate construct $(P=0.0071)$ and $61 \%$ less irreversible deformation than the PFLP without kickstand construct $(P=0.0014)$. The blade plate was the only group that had significantly less irreversible deformation $(59 \%)$ than the PFLP with the kickstand construct $(P=0.0093)$. No significant difference was detected between the blade plate and the PFLP with kickstand. During loading to failure, all constructs failed at the osteotomy site with irreversible bending of the implant and collapse of the medial fracture gap. Of note, none of the lateral fracture gaps had bone-to-bone contact during or at the end of testing. None of the constructs had screw or implant fracture or screw or implant pullout.

\section{DISCUSSION}

As interest in minimally invasive plating techniques increases, a percutaneous plating solution for subtrochanteric 


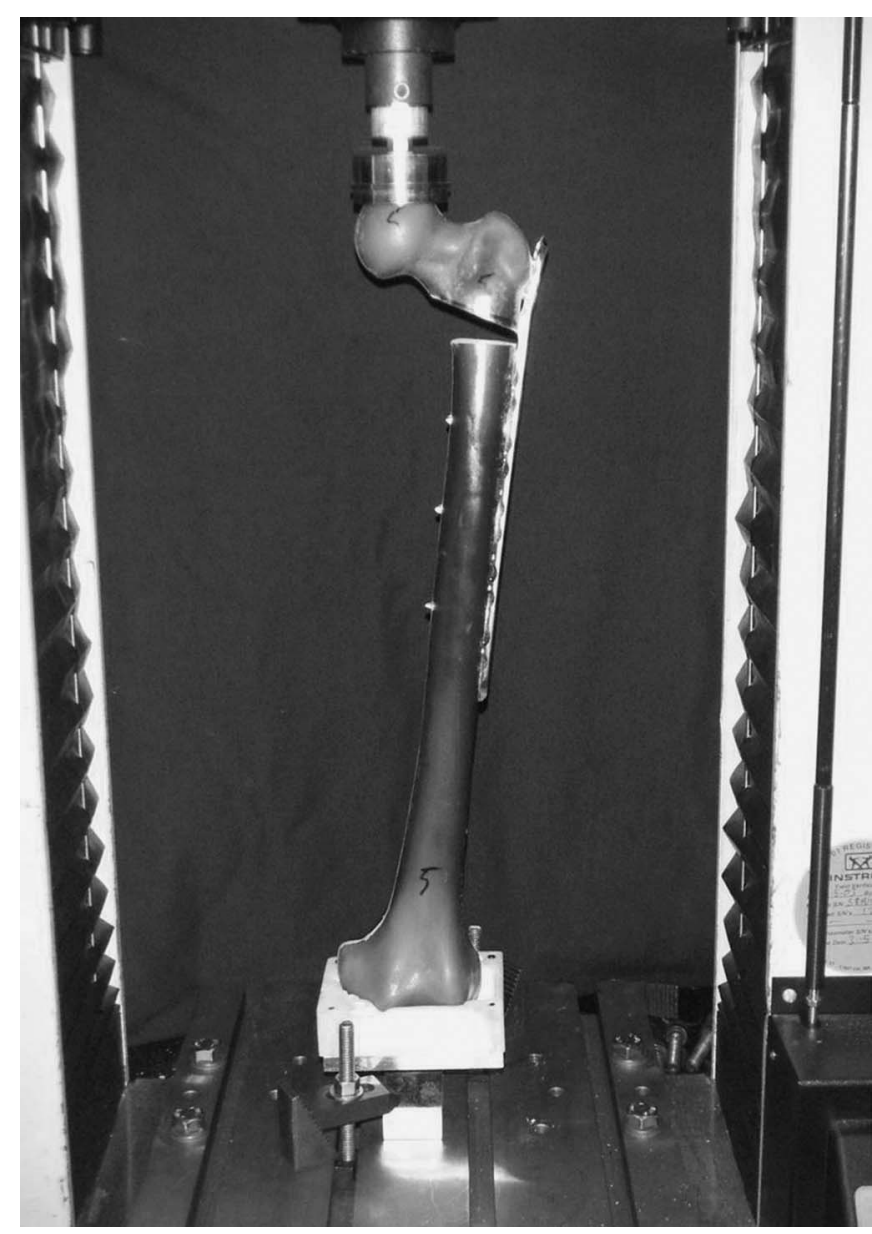

FIGURE 2. Axial stiffness and cyclic loading to failure model.

femur fractures provides an attractive option. No previously published biomechanical studies have compared newer locking plate technology, which allows for percutaneous insertion, with the angled blade plate for subtrochanteric femur fractures. This study shows that locking plates can be comparable and, in some instances (PFLP with kickstand), can provide more axial stiffness than the angled blade plate.

There are few published biomechanical studies comparing the angled blade plate with nonlocked extramedullary devices in a subtrochanteric fracture model. ${ }^{48}$ Most of the biomechanical studies available have compared nonlocked extramedullary devices with intramedullary devices. ${ }^{2,4,5,15} \mathrm{Few}$ have actually included the angled blade plate. Although there are biomechanical data for subtrochanteric femur fracture models, no other published study has compared the devices used in this study, this specific unstable fracture model with extramedullary devices, and the specific testing protocols used. Therefore, comparing our results with previously published biomechanical studies would not be valid.

The limitations of this study are those that are similar to all biomechanical studies. Although synthetic femurs may not ideally recreate the in vivo environment, the third-generation synthetic composite Sawbones have been shown to be similar to human cortical bone in axial and torsional stiffness and also

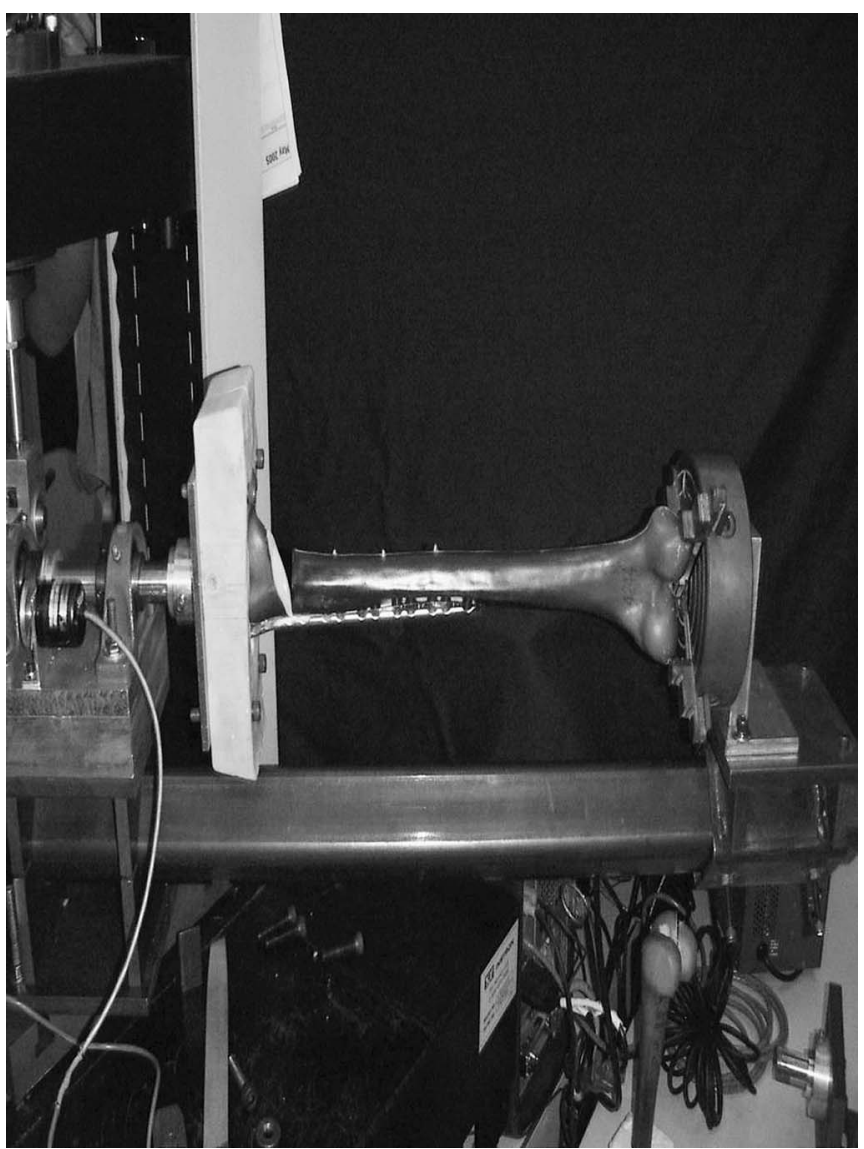

FIGURE 3. Torsional stiffness model.

feature decreased variability, which enhances statistical resolving power. ${ }^{40,41}$ Similarly in the clinical situation, fracture reduction is a key component for construct stiffness. By creating the osteotomy after plate fixation, we attempted to remove fracture reduction as a variable that could affect the biomechanical testing. Although this did not recreate the clinical situation, it did create uniformity in the constructs and allowed us to evaluate the biomechanical properties of the different implants.

The PFLP with the kickstand screw provided comparatively greater axial stiffness, less torsional stiffness, and equivalent irreversible deformation in cyclic loading when compared with the blade plate. The kickstand screw adds important axial stability but has no significant role in

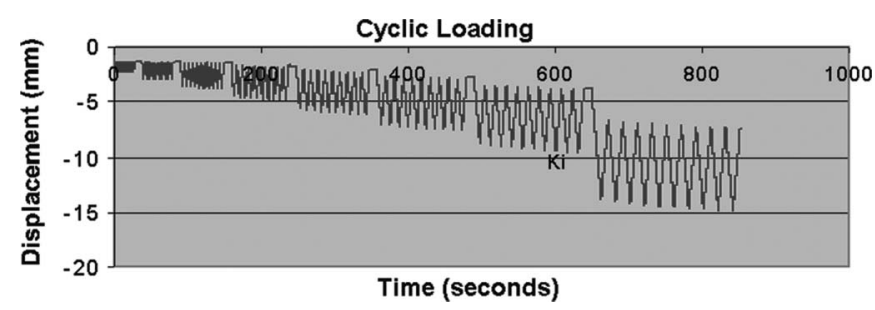

FIGURE 4. Cyclic axial loading time-displacement graph showing irreversible deformation for the PFLP with kickstand screw. 
TABLE 2. Axial Stiffness, Torsional Stiffness, and Irreversible Deformation Due to Cyclic Loading Versus Plate Design

\begin{tabular}{lllc}
\hline & $\begin{array}{c}\text { Axial Stiffness } \\
(\mathbf{N} / \mathbf{m m})\end{array}$ & $\begin{array}{c}\text { Torsional } \\
\text { Stiffness } \\
(\mathbf{N m} / \text { degree) }\end{array}$ & $\begin{array}{c}\text { Cyclic Loading } \\
\text { Irreversible } \\
\text { Deformation }(\mathbf{m m})\end{array}$ \\
\hline Blade plates (control) & $43.6 \pm 3.7$ & $2.42 \pm 0.08^{*}$ & $9.5 \pm 2.7$ \\
Broad plates & $29.8 \pm 8.9 \dagger$ & $1.60 \pm 0.11$ & $13.2 \pm 2.7 \ddagger$ \\
PFLP kickstand & $92.2 \pm 17.4 \S$ & $1.89 \pm 0.39$ & $6.3 \pm 1.2$ \\
PFLP no kickstand & $47.6 \pm 12.6$ & $1.76 \pm 0.12$ & $16.1 \pm 4.59$ \\
\hline
\end{tabular}

Values are expressed as mean $\pm \mathrm{SD}$.

* Significantly different from broad plates $(P<0.0001)$ and PFLP with $(P=0.0017)$ and without $(P=0.0002)$ the kickstand.

†Significantly different from PFLP with $(P<0.0001)$ and without $(P=0.03)$ the kickstand.

$\ddagger$ Significantly different from PFLP with the kickstand $(P=0.0071)$.

§Significantly different from control (blade plates, $P<0.0001)$, broad plates $(P<$ $0.0001)$, and PFLP without kickstand $(P<0.0001)$

TSignificantly different from control (blade plates, $P=0.0093$ ) and PFLP with the kickstand $(P=0.0014)$.

contributing to torsional stiffness. The PFLP with the kickstand screw is biomechanically equivalent to the angled blade plate, but it allows for percutaneous insertion and avoids the potential morbidity accompanying an extensile lateral approach to the femur. Further studies are required to determine if these biomechanical data are reflective of clinical outcomes.

\section{ACKNOWLEDGMENTS}

The authors thank Synthes for providing the implants and composite femurs for this study. The authors also thank $M$. M. Manring, PhD, for his editorial efforts in preparing this article.

\section{REFERENCES}

1. Lechner JD, Rao JP, Stashak G, et al. Subtrochanteric fractures. A retrospective analysis. Clin Orthop Relat Res. 1990;259:140-145.

2. Kummer FJ, Olsson O, Pearlman CA, et al. Intramedullary versus extramedullary fixation of subtrochanteric fractures. A biomechanical study. Acta Orthop Scand. 1998;69:580-584.

3. Brien WW, Wiss DA, Becker V Jr, et al. Subtrochanteric femur fractures: a comparison of the Zickel nail, 95 degrees blade plate, and interlocking nail. J Orthop Trauma. 1991;5:458-464.

4. Tencer AF, Johnson KD, Johnston DW, et al. A biomechanical comparison of various methods of stabilization of subtrochanteric fractures of the femur. J Orthop Res. 1984;2:297-305.

5. Curtis MJ, Jinnah RH, Wilson V, et al. Proximal femoral fractures: a biomechanical study to compare intramedullary and extramedullary fixation. Injury. 1994;25:99-104.

6. Sadowski C, Lubbeke A, Saudan M, et al. Treatment of reverse oblique and transverse intertrochanteric fractures with use of an intramedullary nail or a 95 degrees screw-plate: a prospective, randomized study. $J$ Bone Joint Surg Am. 2002;84-A:372-381.

7. van Meeteren MC, van Rief YE, Roukema JA, et al. Condylar plate fixation of subtrochanteric femoral fractures. Injury. 1996;27:715-717.

8. Miedel R, Ponzer S, Tornkvist H, et al. The standard Gamma nail or the Medoff sliding plate for unstable trochanteric and subtrochanteric fractures. A randomised, controlled trial. J Bone Joint Surg Br. 2005; $87: 68-75$

9. Boldin C, Seibert FJ, Fankhauser F, et al. The proximal femoral nail (PFN) - a minimal invasive treatment of unstable proximal femoral fractures: a prospective study of 55 patients with a follow-up of 15 months. Acta Orthop Scand. 2003;74:53-58.
10. Saarenpaa I, Heikkinen T, Jalovaara P. Treatment of subtrochanteric fractures. A comparison of the Gamma nail and the dynamic hip screw: short-term outcome in 58 patients. Int Orthop. 2007;31:65-70.

11. Cheng MT, Chiu FY, Chuang TY, et al. Treatment of complex subtrochanteric fracture with the long gamma AP locking nail: a prospective evaluation of 64 cases. $J$ Trauma. 2005;58:304-311.

12. Starr AJ, Hay MT, Reinert CM, et al. Cephalomedullary nails in the treatment of high-energy proximal femur fractures in young patients: a prospective, randomized comparison of trochanteric versus piriformis fossa entry portal. J Orthop Trauma. 2006;20:240-246.

13. Neher C, Ostrum RF. Treatment of subtrochanteric femur fractures using a submuscular fixed low-angle plate. Am J Orthop. 2003;32:29-33.

14. Kregor PJ, Obremskey WT, Kreder HJ, et al. Evidence-Based Orthopaedic Trauma Working Group. Unstable pertrochanteric femoral fractures. J Orthop Trauma. 2005;19:63-66.

15. Bredbenner TL, Snyder SA, Mazloomi FR, et al. Subtrochanteric fixation stability depends on discrete fracture surface points. Clin Orthop Relat Res. 2005;432:217-225.

16. Mahomed MN, Harrington IJ, Hearn TC. Biomechanical analysis of the Medoff sliding plate. J Trauma. 2000;48:93-100.

17. Tornetta P III. Subtrochanteric femur fracture. J Orthop Trauma. 2002;16: 280-283.

18. Yoo MC, Cho YJ, Kim KI, et al. Treatment of unstable peritrochanteric femoral fractures using a 95 degrees angled blade plate. J Orthop Trauma. 2005; 19:687-692.

19. Siebenrock K, Muller U, Ganz R. Indirect reduction with a condylar blade plate for osteosynthesis of subtrochanteric femoral fractures. Injury. 1998; 29:S-C7-S-C15.

20. Kinast C, Bolhofner BR, Mast JW, et al. Subtrochanteric fractures of the femur. Results of treatment with the 95 degrees condylar blade-plate. Clin Orthop Relat Res. 1989;238:122-130.

21. Vaidya SV, Dholakia DB, Chatterjee A. The use of a dynamic condylar screw and biological reduction techniques for subtrochanteric femur fracture. Injury. 2003;34:123-128.

22. Ali M. Treatment of comminuted subtrochanteric fractures by dynamic hip screw. J Pak Med Assoc. 1995;45:212-215.

23. Blatter G, Janssen M. Treatment of subtrochanteric fractures of the femur: reduction on the traction table and fixation with dynamic condylar screw. Arch Orthop Trauma Surg. 1994;113:138-141.

24. Celebi L, Can M, Muratli HH, et al. Indirect reduction and biological internal fixation of comminuted subtrochanteric fractures of the femur. Injury. 2006;37:740-750.

25. Hsu YT, Wu CC, Su CY, et al. Indirect reduction with sliding compression screw stabilization for subtrochanteric fractures. Chang Gung Med J. 2006;29:190-197.

26. Lee PC, Yu SW, Hsieh PH, et al. Bridge-plating osteosynthesis of 20 comminuted subtrochanteric fractures with dynamic hip screw. Chang Gung Med J. 2002;25:803-810.

27. Pai CH. Dynamic condylar screw for subtrochanteric femur fractures with greater trochanteric extension. J Orthop Trauma. 1996;10:317-322.

28. Haidukewych GJ, Israel TA, Berry DJ. Reverse obliquity fractures of the intertrochanteric region of the femur. J Bone Joint Surg Am. 2001;83-A: 643-650.

29. Vanderschot P, Vanderspeeten K, Verheyen L, et al. A review on 161 subtrochanteric fractures - risk factors influencing outcome: age, fracture pattern and fracture level. Unfallchirurg. 1995;98:265-271.

30. Ricci WM, Devinney S, Haidukewych G, et al. Trochanteric nail insertion for the treatment of femoral shaft fractures. J Orthop Trauma. 2005;19: 511-517.

31. Hesse B, Gächter A. Complications following the treatment of trochanteric fractures with the gamma nail. Arch Orthop Trauma Surg. 2004;124:692-698.

32. Wolinsky P, Tejwani N, Richmond JH, et al. Controversies in intramedullary nailing of femoral shaft fractures. Instr Course Lect. 2002;51:291-303.

33. Kraemer WJ, Hearn TC, Powell JN, et al. Fixation of segmental subtrochanteric fractures. A biomechanical study. Clin Orthop Relat Res. 1996;332:71-79.

34. Rantanen J, Aro HT. Intramedullary fixation of high subtrochanteric femoral fractures: a study comparing two implant designs, the gamma nail and the intramedullary hip screw. J Orthop Trauma. 1998;12:249-252.

35. Madsen JE, Naess L, Aune AK, et al. Dynamic hip screw with trochanteric stabilizing plate in the treatment of unstable proximal femoral 
fractures: a comparative study with the gamma nail and compression hip screw. J Orthop Trauma. 1998;12:241-248.

36. Kang S, McAndrew MP, Johnson KD. The reconstruction locked nail for complex fractures of the proximal femur. J Orthop Trauma. 1995;9:453-463.

37. Ricci WM, Bellabarba C, Evanoff B, et al. Retrograde versus antegrade nailing of femoral shaft fractures. J Orthop Trauma. 2001;15:161-169.

38. Kesemenli C, Subasi M, Necmioglu S, et al. Treatment of multifragmentary fractures of the femur by indirect reduction (biological) and plate fixation. Injury. 2002;33:691-699.

39. Krettek C, Schandelmaier P, Miclau T, et al. Minimally invasive percutaneous plate osteosynthesis (MIPPO) using the DCS in proximal and distal femoral fractures. Injury. 1997;28:A20-A30.

40. Cristofolini L, Viceconti M, Cappello A, et al. Mechanical validation of whole bone composite femur models. J Biomech. 1996;29:525-535.

41. Heiner AD, Brown TD. Structural properties of a new design of composite replicate femurs and tibias. $J$ Biomech. 2001;34:773-781.

42. Wheeler DL, Croy TJ, Woll TS, et al. Comparison of reconstruction nails for high subtrochanteric femur fracture fixation. Clin Orthop Relat Res. 1997;338:231-239.
43. Fracture and dislocation compendium. Orthopaedic Trauma Association Committee for Coding and Classification. J Orthop Trauma. 1996;10: $1-154$.

44. Cordey J, Borgeaud M, Frankle M, et al. Loading model for the human femur taking the tension band effect of the ilio-tibial tract into account. Injury. 1999;30:A26-A30.

45. Mahomed N, Harrington I, Kellam J, et al. Biomechanical analysis of the gamma nail and sliding hip screw. Clin Orthop Relat Res. 1994;304: 280-288.

46. Zlowodzki M, Williamson S, Cole PA, et al. Biomechanical evaluation of the less invasive stabilization system, angled blade plate, and retrograde intramedullary nail for the internal fixation of distal femur fractures. J Orthop Trauma. 2004;18:494-502.

47. Marti A, Fankhauser C, Frenk A, et al. Biomechanical evaluation of the less invasive stabilization system for the internal fixation of distal femur fractures. J Orthop Trauma. 2001;15:482-487.

48. Lundy DW, Acevedo JI, Ganey TM, et al. Mechanical comparison of plates used in the treatment of unstable subtrochanteric femur fractures. J Orthop Trauma. 1999;13:534-538. 